\title{
Acceptance and Attitude toward COVID-19 Vaccination among the Public in Saudi Arabia: A Cross-sectional Study
}

\author{
Eyad M Alduwayghiri ${ }^{1}$, Nubesh Khan ${ }^{2}$
}

\begin{abstract}
Aim and objective: This cross-sectional study aimed to investigate vaccine acceptance and attitude to novel coronavirus disease 2019 (COVID-19) vaccine among the general population in Saudi Arabia.

Materials and methods: The present study was conducted among the general population in Saudi Arabia above 18 years of age. The data were collected online, by questionnaire containing 20 questions using Google form, in both Arabic and English, and were distributed to friends, families, and relatives through mail, WhatsApp groups, and Twitter. The questionnaire was randomly given out to the people living in five provinces of Saudi Arabia.

Results: A sum of 1,713 participants responded to the online survey. The greatest number of participants were at the age between 31 years and 45 years $(36.6 \%)$ and $56.4 \%$ were females. The results showed that $74.6 \%$ have not registered for the vaccination and $44.9 \%$ responded not to take the vaccine. About $60.80 \%$ were concerned about the side effects, and $48.2 \%$ were worried about the allergic reaction to COVID-19 vaccine. About $49.1 \%$ of participants agreed that preventive measures, such as wearing mask, social distance, and using sanitizers, keep the virus away, and $53.20 \%$ believes that being exposed to disease naturally is safer for the immune system. Around $23.90 \%$ believes that vaccination cannot reduce the possibility of getting COVID-19.

Conclusion: In the present study, the negative attitudes and afraid of the side effects of the vaccine are the most reasons for indecision and rejection about COVID-19 vaccine. For the future COVID-19 vaccination campaign not to fail, the Ministry of Saudi Arabia should closely follow the vaccination development processes, inform the public transparently, and consider public's concerns.

Clinical significance: The results of the current study will help the Ministry of Health in Saudi Arabia to attempt proactive missions by featuring the significance of immunization to the population and empowering vaccine take-up and acceptance.

Keywords: Acceptance, Attitude, COVID-19, Saudi Arabia, Vaccination.

The Journal of Contemporary Dental Practice (2021): 10.5005/jp-journals-10024-3114
\end{abstract}

\section{INTRODUCTION}

Coronavirus disease-2019 (COVID-19) infection, which the World Health Organization (WHO) has declared a global pandemic, caused a widespread impact on health, including substantial mortality, repercussions for the global economy caused by physical distancing measures, with the greatest consequences for the most vulnerable in society. ${ }^{1}$ Since the first case of COVID-19 in Saudi Arabia was detected on Monday, March 2, 2020, the Saudi Ministry of Health vigilantly monitored the situation and developed country-specific measures, like social distancing, hand washing, wearing masks, and lockdown procedures, in line with the WHO guidelines in dealing with the outbreak. ${ }^{2}$

The long-term success of the public prevention efforts to the COVID-19 will depend on acquired immunity in a sufficient proportion of the population (herd immunity), which is estimated to be $67 \%$ for COVID-19. ${ }^{3}$ The vaccines are known to be effective in creating a long-lasting immune memory to control and prevent the disease and reducing the ongoing health crisis. ${ }^{4}$ In response, rapid global efforts were made to develop and test vaccines against SARS-CoV-2. Vaccines typically require years of research and testing before reaching the clinic, but in 2020, scientists embarked on a race to produce safe and effective coronavirus vaccines in record time. More than 60 vaccines are still going through a three-stage clinical trial process that is required before they are sent to regulatory agencies for approval. Given the urgent need, some vaccines like Pfizer/BioNtech, Moderna,

\footnotetext{
${ }^{1,2}$ Department of Preventive Dentistry, College of Dentistry in Ar Rass, Qassim University, Saudi Arabia

Corresponding Author: Eyad M Alduwayghiri, Department of Preventive Dentistry, College of Dentistry in Ar Rass, Qassim University, Saudi Arabia, e-mail: ey.alduwayghiri@qu.edu.sa

How to cite this article: Alduwayghiri EM, Khan N. Acceptance and Attitude toward COVID-19Vaccination among the Public in Saudi Arabia: A Cross-sectional Study. J Contemp Dent Pract 2021;22(7):730-734.

Source of support: Nil

Conflict of interest: None
}

and Oxford/Astra Zeneca have been introduced in the market by compressing the clinical process for SARS-CoV- 2 and running trial phases simultaneously. ${ }^{5}$

Despite the extraordinary speed of scientific discovery regarding COVID-19, there are still a lot of uncertainties and concerns on novel vaccines in terms of safety and effectiveness. A recent global survey suggested that nearly $30 \%$ of participants hesitate to take a COVID-19 vaccine when it becomes available. ${ }^{6}$ The unwillingness and uncertainty about receiving a COVID-19 vaccine will be a significant challenge in achieving the vaccination coverage required for population immunity. Concerns identified to date for intending not to receive the COVID-19 vaccine include worries about the newness and safety of the vaccine as well as about potential side effects. ${ }^{7,8}$ 
Saudi Arabia launched its coronavirus vaccination campaign on December 17, 2020, after receiving the first shipment of the Pfizer-BioNTech vaccine. ${ }^{9}$ The people were asked to register for the vaccine through the "SEHATY" (MY HEALTH) online application and were made free for the citizens and residents in Saudi Arabia. Henceforth, it is significant to investigate the acknowledgment of COVID-19 vaccines and their indicators just as perspectives toward COVID-19 vaccines among people in Saudi Arabia. The aim of the present study is to assess the vaccine acceptance and attitude to novel COVID-19 vaccine among the general population in Saudi Arabia.

The results of the current study could assist the Ministry of Health in Saudi Arabia to attempt proactive missions and very much planned techniques by featuring the significance of immunization to the population and empowering vaccine take-up and acceptance, to improve the immunity furthermore, and to restrict the spread of the pandemic.

\section{Materials and Methods}

\section{Study Design and Participants}

The present cross-sectional study was conducted among the general population of Saudi Arabia, from January 7, 2021, to January 26, 2021. The data were collected online, by questionnaire using Google form, in both Arabic and English, and were distributed to friends, families, and relatives through mail, WhatsApp groups, and Twitter. The background and objectives of the study were mentioned in the beginning of the questionnaire and were informed that all the information's provided would be anonymous and confidential. The questionnaire was randomly distributed to the people living in five provinces of Saudi Arabia. People aged 18 years or older who can understand the content of the questionnaire and who agreed to participate in the study were asked to complete the questionnaire and online informed consent was obtained before proceeding with the questionnaire.

\section{Study Instrument}

The questionnaire to be used in the research was prepared based on the literature review and discussion by the researchers and there are 20 questions in total. The questionnaire contains seven questions for sociodemographic characteristics (gender, age, occupation, etc.) and questions for health conditions. Respondents were asked to inform if they were tested for COVID-19 or any one of their family members was infected with COVID-19. The attitudes and perceptions toward COVID-19 vaccine were assessed by asking 10 questions. The questionnaire required an expected time of $2-3$ minutes to finish.

\section{Ethical Considerations}

The study was approved by the Ethical Committee, (DRC/0020FA/21) Ar Rass Dental College, Qassim University, Saudi Arabia.

\section{Statistical Analysis}

The data analysis was performed using Microsoft Excel 2019 and SPSS version 25.0 (Chicago, Illinois, USA). Microsoft Excel was used for editing, sorting, and coding. The excel file was then imported into SPSS software. Descriptive statistics were calculated for demographic factors and answers toward attitudes toward COVID-19 vaccine.

\section{Results \\ Study Population}

The study was limited for a period of 3 weeks and till that duration, 1,728 people responded and in that $15(0.9 \%)$ respondents did not give the informed consent and failed to complete the questionnaire and were excluded and the data were calculated from 1,713 (99.13\%) participants.

The greatest number of participants were at the age between 31 years and 45 years (36.6\%) and more than half of them $(56.4 \%)$ were females. Nearly all respondents (>98\%) were Saudi citizens and most of the participants (53.10\%) were from the central province.

Of the participants, $85.30 \%$ were nonhealth workers and $75.3 \%$ does not have any health problems, $11.8 \%$ are diabetics, $10.1 \%$ are hypertensive, $7.6 \%$ with respiratory problems, and $2.5 \%$ with cardiac problems. Detailed sociodemographic characteristics of participants are in Table 1.

Almost half of the participants (49\%) have done the COVID-19 test, only $9.2 \%$ of them were positive for COVID-19, and $55.5 \%$ of respondents reported that none of their family members were infected with COVID-19 (Fig. 1).

\section{Attitudes toward Novel COVID-19 Vaccine}

The COVID-19 vaccine was made free for all the people in Saudi Arabia and the ministry opened an online application, in which the people must register for the vaccination. The people were encouraged to take the vaccine but was not made compulsory.

Table 1: Demographic details of study participants

\begin{tabular}{|c|c|}
\hline \multicolumn{2}{|l|}{ Age (years) } \\
\hline $18-30$ & $29.25 \%$ \\
\hline $31-45$ & $36.60 \%$ \\
\hline $46-60$ & $19.20 \%$ \\
\hline $61-75$ & $13.06 \%$ \\
\hline Above 76 & $1.89 \%$ \\
\hline \multicolumn{2}{|l|}{ Gender } \\
\hline Male & $43.60 \%$ \\
\hline Female & $56.40 \%$ \\
\hline \multicolumn{2}{|l|}{ Nationality } \\
\hline Saudi & $98.2 \%$ \\
\hline Non-Saudi & $1.80 \%$ \\
\hline \multicolumn{2}{|l|}{ Educational level } \\
\hline Doctorate & $15 \%$ \\
\hline Masters & $39 \%$ \\
\hline Bachelors & $29 \%$ \\
\hline High school & $11 \%$ \\
\hline Others & $6 \%$ \\
\hline \multicolumn{2}{|l|}{ Profession } \\
\hline Health workers & $14.70 \%$ \\
\hline Nonhealth workers & $85.30 \%$ \\
\hline \multicolumn{2}{|l|}{ Province } \\
\hline Central & $53.10 \%$ \\
\hline Eastern & $21.29 \%$ \\
\hline Western & $14.22 \%$ \\
\hline Northern & $9.06 \%$ \\
\hline Southern & $2.88 \%$ \\
\hline
\end{tabular}




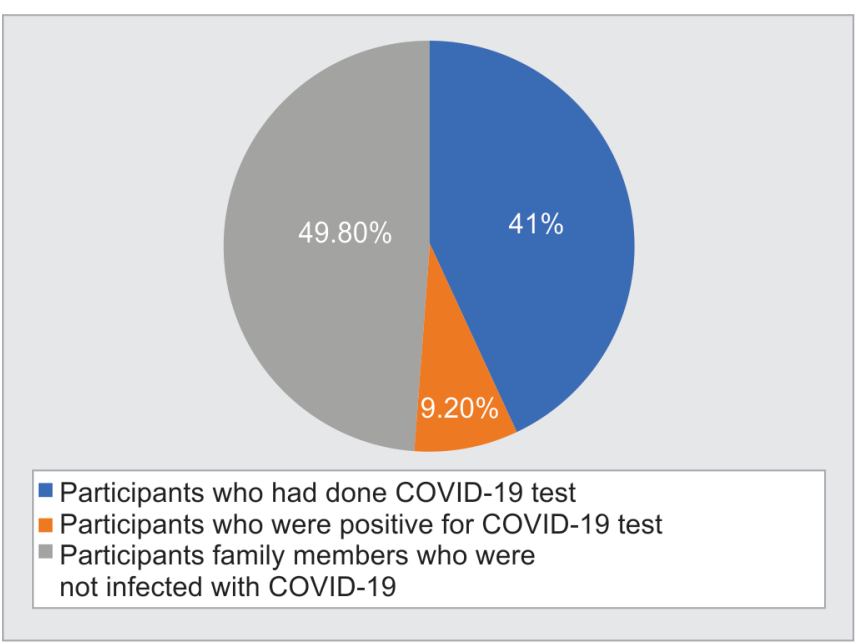

Fig. 1: COVID-19 test

When asked about the registration for vaccination, it was found that $74.6 \%$ (almost three-quarter) have not registered for the vaccination yet (Fig. 2), and $44.9 \%$ responded that they prefer not to take the vaccine while $55.1 \%$ of participants agreed to take the vaccine, even if it is not mandatory.

About $60.80 \%$ of respondents were concerned about the side effects of COVID-19 vaccine in future, $61 \%$ were scared of injection, and $48.2 \%$ were worried about the allergic reaction to COVID-19 vaccine.

Almost half $(49.1 \%)$ of participants agreed that vaccination is not needed as preventive measures, such as wearing mask, keeping social distance, and using sanitizers, keep the virus away, and $53.20 \%$ believe that being exposed to disease naturally is safer for the immune system than being exposed through vaccination. In addition to this, $23.90 \%$ believes that vaccination cannot reduce the possibility of getting COVID-19 and $48.39 \%$ is not sure about the duration of protection after being vaccinated, so have not decided about taking vaccination.

Moreover, the results of this study showed that $55.70 \%$ of participants are not confident in vaccine as it is new and produced in a short span of time, and about $44 \%$ agreed that the vaccine will not be effective for those who are already infected with COVID-19. Figure 3 shows the response for the questionnaire toward COVID-19 vaccine.

\section{Discussion}

COVID-19 is an emerging infectious disease that poses a significant threat to public health. There is an urgent and important need to manufacture and distribute enough safe and effective vaccine to immunize individuals to protect the entire global community from the threat of morbidity and mortality from COVID-19 infection. ${ }^{10}$

The importance of knowledge about vaccines and attitude toward vaccination has become essential in the previous few months. This is the first kind of study done in Saudi Arabia to assess the attitude of the public toward the novel COVID-19 vaccine after the introduction of vaccine. Our study investigated the attitude toward COVID-19 vaccination in a population of 1,698 respondents, mainly from the central province. While this is a limited sample size, these data may be considered representative.

In the present study, most of the participants were between 31 years and 45 years of age (36.6\%) and $56.4 \%$ were females. Our

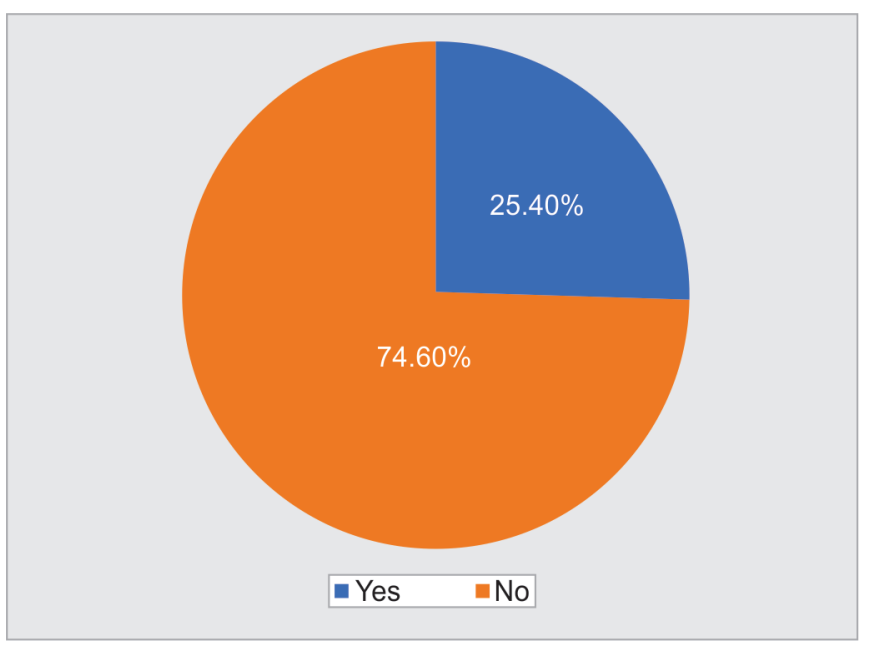

Fig. 2: Registration for COVID-19 vaccine

study showed that $76.40 \%$ of respondents did not register for the vaccination, and $44.9 \%$ are not willing to take the vaccine and $55.1 \%$ of participants agreed to take the vaccine.

Though there have been limited studies to explore the intention to uptake the COVID-19 vaccine in the current crisis, the study conducted by Elimat et al. ${ }^{11}$ also showed that public adequacy of COVID-19 immunizations was genuinely low (37.4\%) in Jordan.

Fue et al. ${ }^{12}$ reported that $72.5 \%$ of Chinese general population's intention to uptake COVID-19 vaccine and Thunstrom et al. ${ }^{13}$ who conducted a study in the United States reported $80 \%$ acceptance of the COVID-19 vaccine among the study population.

A survey based on a representative from 19 countries involving 13,426 contributors showed that the worldwide acquiring of COVID-19 immunizations is less than $54.80 \%$ from Russia to more than $88.60 \%$ from China. ${ }^{14}$

Vaccine history was the most important predictor of the intent to receive a COVID-19 vaccine, a factor that reflects confidence in vaccines in general. The low acknowledgment level of COVID-19 vaccines among people in Saudi can be due to different causes, some of which are imparted to the wide worldwide group.

The current study confirmed about the negative attitudes toward vaccination such as side effects of vaccine and worried about getting to be injected. This is in accordance with Pogue et al. discovery, where large portions of contributors (63\%) in the USA expressed that they were concerned over the symptoms of the COVID-19 vaccines. ${ }^{15}$ Studies conducted by Palamenghi et al. ${ }^{16}$ and Wang et al. ${ }^{17}$ suggest that the level of reluctance to vaccinate against COVID-19 is higher than vaccine hesitancy for usual vaccines in several countries.

As the vaccine is new and produced in a short span of time, $55.70 \%$ of participants in the current study are not confident and $44 \%$ believes that the vaccine will not be effective for those who are already infected with COVID-19. The present study showed that $48.39 \%$ are not sure about the duration of protection after being vaccinated and $23.90 \%$ believes that vaccination cannot reduce the possibility of getting COVID-19.

The pace of COVID-19 vaccine improvement and enlistment in under a year may have played a role in decreasing the acquiring level. The speed of antibody improvement and enlistment in under a year may have interceded a job in bringing down the acknowledgment level. 


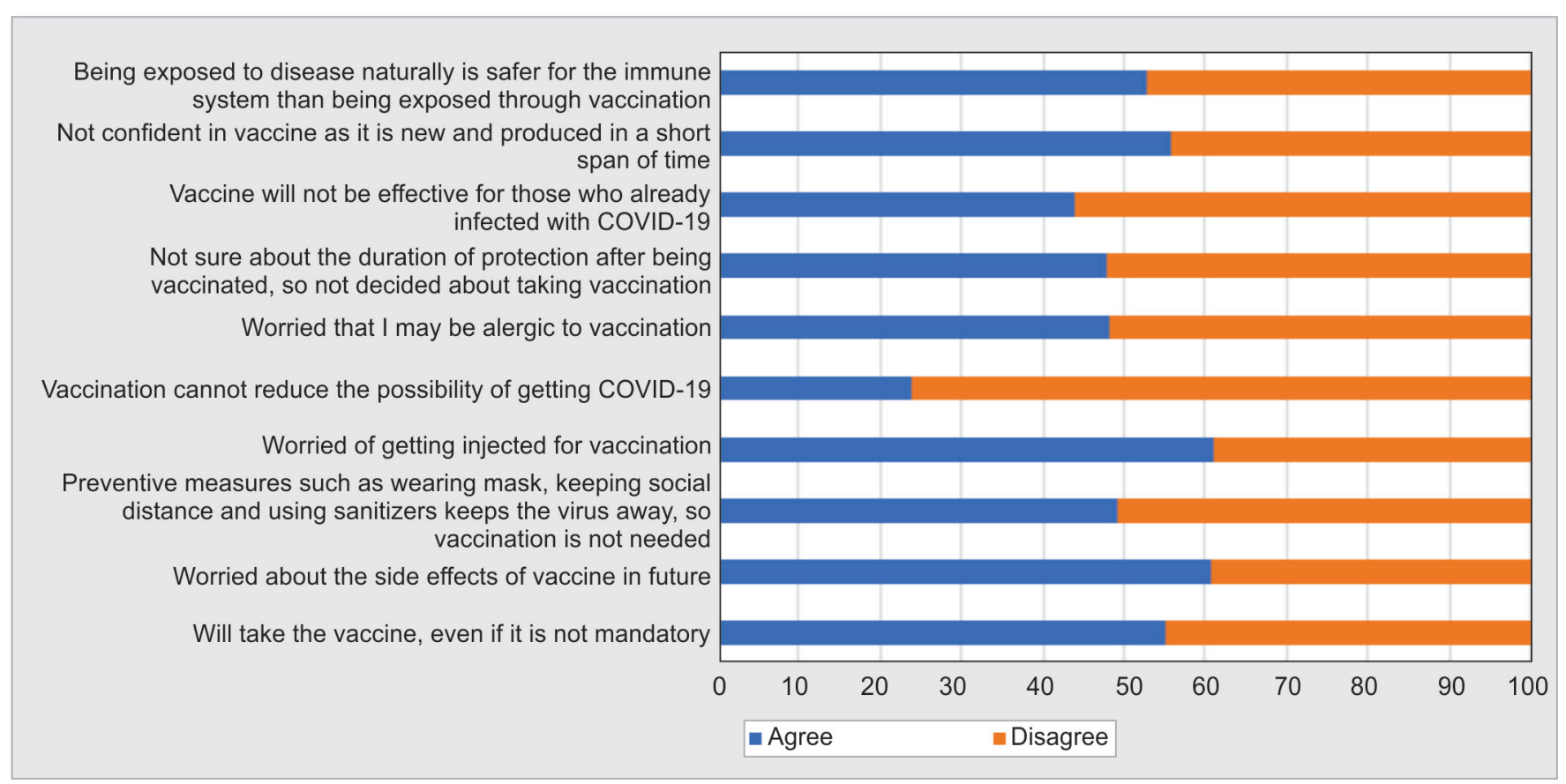

Fig. 3: Attitudes toward COVID-19 vaccine

Anotherworldwide phenomenon that added to a lowacceptance level is the various missions dispatched by antivaccinationists. Such missions via web-based media with manufactured, bogus, and at times misdirecting Arabic interpretations feed the planned belief of specific individuals.

It is pivotal to disperse straight forward and precise data about vaccines' protection and adequacy to acquire the faith of the people particularly the reluctant and incredulous ones. ${ }^{18}$ Hence, acquiring an understanding of the assets that individuals trust the most to get data about COVID-19 vaccines is basic for the achievement of any future public vaccination campaign.

As alternatives to vaccines, $49.1 \%$ of respondents in our study preferred general "natural" methods, such as wearing mask, keeping social distance, and using sanitizers, keep the virus away and $53.20 \%$ believe that being exposed to disease naturally is safer for the immune system than being exposed through vaccination.

While these are general preventive measures, the lockdown caused by the coronavirus pandemic and the ensuing fatigue proves that these measures work only for a limited amount of time before negatively impacting society. ${ }^{19}$

The current pandemic entails three important challenges for public confidence in and uptake of a future, licensed vaccine. First, the evidence showed that if a new vaccine is introduced, the level of hesitancy will be high. ${ }^{20}$ Second, the people's trust over the vaccine will take time, and finally, the challenge against the misinformation by antivaccination campaigners. ${ }^{21}$

The major limitation of the study was the short duration, so as to know the public's initial attitude toward COVID-19 vaccine. After the survey was finished and at the time of submission of the manuscript, we found an increased awareness among the public and more people were accepting the COVID-19 vaccine. The reason for the sudden change in attitude toward vaccine was due to great effort by the Ministry of Health, Saudi Arabia, in spreading most valid data's about COVID-19 vaccines, planned involvement in terms of alertness movements through a wide range of interactive media to circulate more straightforward data about the protection and potency of the vaccines, opened more vaccination centers, drive through vaccinations, and even made vaccine available at the doorsteps for the bedridden peoples.

\section{Conclusion}

Throughout this study, we found an initial reluctance toward COVID-19 vaccine among public people in Saudi Arabia. Investigating sociodemographic and psychosocial factors that affect vaccine acceptance is fundamental for an effective immunization plan. In the meantime, an efficacious vaccine has the potential to have a major impact on the pandemic if used in populations at the proper time.

\section{ACKNOWLedgment}

Researchers would like to thank the Deanship of Scientific Research, Qassim University for funding the publication of this project.

\section{References}

1. Zhou F, Yu T, Du R, et al. Clinical course and risk factors for mortality of adult inpatients with COVID-19 in Wuhan, China: a retrospective cohort study. Lancet 2020;395(10229):1054-1062. DOI: 10.1016/S01406736(20)30566-3.

2. Al-Hanawi MK, Angawi K, Alshareef N, et al. Knowledge, attitude and practice toward COVID-19 among the public in the Kingdom of Saudi Arabia: a cross-sectional study. Front Public Health 2020;8:217. DOI: 10.3389/fpubh.2020.00217.

3. Randolph HE, Barreiro LB. Herd immunity: understanding COVID-19. Immunity 2020;52(5):737-741. DOI: 10.1016/j.immuni.2020.04.012.

4. Bish A, Yardley L, Nicoll A, et al. Factors associated with uptake of vaccination against pandemic influenza: a systematic review. Vaccine 2011;29(38):6472-6484. DOI: 10.1016/j.vaccine.2011.06.107.

5. Wang Z, Schmidt F, Weisblum $Y$, et al. mRNA vaccine-elicited antibodies to SARS-CoV-2 and circulating variants. Nature 2021:1-7. DOI: 10.1038/s41586-021-03324-6. 
6. Kwok KO, Lai F, Wei WI, et al. Herd immunity-estimating the level required to halt the COVID-19 epidemics in affected countries. J Infect 2020;80(6):e32-e33. DOI: 10.1016/j.jinf.2020.03.027.

7. Neumann-Böhme S, Varghese NE, Sabat I, et al. Once we have it, will we use it? A European survey on willingness to be vaccinated against COVID-19. Eur J Health Econ 2020;21(7):977-982. DOI: 10.1007/s10198020-01208-6.

8. Rhodes $A$, Hoq $M$, Measey MA, et al. Intention to vaccinate against COVID-19 in Australia. Lancet Infect Dis 2020;21(5):e110. DOI: 10.1016/ S1473-3099(20)30724-6.

9. Nasser AA, Alzahrani RM, Fellah CA, et al. Measuring the patients' satisfaction about telemedicine used in Saudi Arabia during COVID-19 pandemic. Cureus 2021;13(2):e13382. DOI: 10.7759/ cureus.13382.

10. Corey L, Mascola JR, Fauci AS, Collins FS. A strategic approach to COVID-19 vaccine R\&D. Science 2020;368(6494):948-950. DOI: 10.1126/science.abc5312.

11. El-Elimat T, Abu Al Samen MM, Almomani BA, et al. Acceptance and attitudes toward COVID-19 vaccines: a cross-sectional study from Jordan. PLoS One 2021;16(4):e0250555. DOI: 10.1371/journal. pone.0250555.

12. Fu C, Wei Z, Pei S, et al. Acceptance and preference for COVID-19 vaccination in health-care workers (HCWs). MedRxiv 2020. DOI: 10.1101/2020.04.09.20060103.

13. Thunstrom $L$, Ashworth M, Finnoff $D$, et al. Hesitancy towards a COVID-19 vaccine and prospects for herd immunity. Available at SSRN 3593098. 2020. DOI: 10.2139/ssrn.3593098.
14. Lazarus JV, Ratzan S, Palayew A, et al. Hesitant or not. A global survey of potential acceptance of a COVID-19 vaccine. Nat Med 2020. DOI: 10.1038/s41591-020-1124-9.

15. Pogue K, Jensen JL, Stancil CK, et al. Influences on attitudes regarding potential COVID-19 vaccination in the United States. Vaccines 2020;8(4):582. DOI: 10.3390/vaccines8040582.

16. Wang K, Wong EL, Ho KF, et al. Intention of nurses to accept coronavirus disease 2019 vaccination and change of intention to accept seasonal influenza vaccination during the coronavirus disease 2019 pandemic: a cross-sectional survey. Vaccine 2020;38(45):70497056. DOI: 10.1016/j.vaccine.2020.09.021.

17. Palamenghi L, Barello S, Boccia S, et al. Mistrust in biomedical research and vaccine hesitancy: the forefront challenge in the battle against COVID-19 in Italy. Eur J Epidemiol 2020;35(8):785-788. DOI: 10.1007/ s10654-020-00675-8.

18. Siegrist $M$, Zingg $A$. The role of public trust during pandemics: implications for crisis communication. Eur Psychol 2014;19(1):23. DOI: 10.1027/1016-9040/a000169.

19. Nitschke JP, Forbes PA, Ali N, et al. Resilience during uncertainty? Greater social connectedness during COVID-19 lockdown is associated with reduced distress and fatigue. $\mathrm{Br} J$ Health Psychol 2020;26(2):553-569. DOI: 10.1111/bjhp.12485.

20. Dubé $E$, Laberge $C$, Guay $M$, et al. Vaccine hesitancy: an overview. Hum Vaccin Immunother 2013;9(8):1763-1773. DOI: 10.4161/hv.24657.

21. Spielmans GI, Parry PI. From evidence-based medicine to marketingbased medicine: evidence from internal industry documents. J Bioethical Inq 2010;7(1):13-29. DOI:_10.1007/s11673-010-9208-8. 